\title{
Stochastic theory for lineshape broadening in quasielastic He atom scattering with interacting adsorbates
}

\author{
R. Martínez-Casado* \\ Lehrstuhl für Physikalische Chemie I, Ruhr-Universität Bochum, D-44801 Bochum, Germany \\ and Instituto de Física Fundamental, Consejo Superior de Investigaciones Científicas, Serrano 123, 28006-Madrid, Spain \\ J. L. Vega, ${ }^{\dagger}$ A. S. Sanz,,$^{\ddagger}$ and S. Miret-Artés ${ }^{\S}$ \\ Instituto de Física Fundamental, Consejo Superior de Investigaciones Científicas, Serrano 123, 28006-Madrid, Spain
}

(Received 17 October 2007; revised manuscript received 14 December 2007; published 10 March 2008)

\begin{abstract}
The activated surface diffusion of interacting adsorbates is described in terms of the so-called interacting single adsorbate approximation, which is applied to the diffusion of $\mathrm{Na}$ atoms on $\mathrm{Cu}(001)$ for coverages up to $20 \%$ in quasielastic He atom scattering experiments. This approximation essentially consists of solving the standard Langevin equation with two noise sources and frictions: a Gaussian white noise accounting for the friction with the substrate, and a white shot noise characterized by a collisional friction simulating the adsorbate-adsorbate collisions. The broadenings undergone by the quasielastic peak are found to be in very good agreement with the experimental data reported at two surface temperatures of 200 and $300 \mathrm{~K}$.
\end{abstract}

DOI: 10.1103/PhysRevB.77.115414

PACS number(s): 68.43.Jk, 05.10.Gg, 68.35.Fx

\section{INTRODUCTION}

The diffusion of atoms, molecules, or small clusters on metal surfaces plays an important role in many technological and industrial applications. Molecular beam epitaxy, heterogeneous catalysis, or the fabrication of nanostructures are examples strongly affected by the kinetics of diffusion. Among the different experimental techniques utilized to analyze the diffusion and adsorbate dynamics, quasielastic helium-atom scattering (QHAS) is a gentle and inert technique commonly applied to fast diffusion. ${ }^{1}$ QHAS has been applied to different metal/metal and adsorbate/metal systems, the diffusion of $\mathrm{Na}$ adatoms (at different coverages) on $\mathrm{Cu}(001)$ being one of the most extensively systems studied so far. ${ }^{2-12}$ At low coverages, this system has been theoretically described within the so-called single adsorbate approximation and analyzed in terms of the motional narrowing effect, ${ }^{4,7}$ and also within the Kramers turnover theory ${ }^{5}$ and the dephasing theory. ${ }^{6}$ In particular, the motional narrowing effect governs the broadening of the lineshapes as a function of the friction, the parallel momentum transfer, the lattice structure, and the jump dynamics. Generalized expressions for the lineshape have been recently given under these different conditions. ${ }^{7,8}$

When dealing with higher coverages, absorbate-adsorbate interactions can no longer be neglected. Then, pairwise interaction potentials are usually introduced into twodimensional Langevin molecular dynamics (LMD) simulations, ${ }^{3}$ where the number of coupled equations to be solved is usually very high (it increases as $2 N$, where $N$ is the number of adparticles considered, typically of the order of 400-500). On the one hand, first, this translates into a high computational cost. Second, the numerical results issued from LMD simulations are difficult to interpret since analytical treatments are not easily implementable. On the other hand, from a physical viewpoint, the broadening (measured as the full width at half maximum) of both the quasielastic $(Q)$ peak ruling the diffusion process and the $T$-mode peaks related to the low frequency motions of the adsorbate (frustrated translational modes) is not well reproduced when compared with the experimental data. ${ }^{3}$ Recently, it has been shown ${ }^{9}$ that good agreement with the experiment can be achieved using simple models where the adsorbate is allowed to also move perpendicular to the surface.

Aimed to provide a theoretical and numerical alternative to the standard procedure at intermediate coverages, we have recently proposed the so-called interacting single adsorbate (ISA) approximation. ${ }^{10-12}$ Within this approach, diffusion is described by using only one standard Langevin equation, which is characterized by the following three contributions: (1) the deterministic, adiabatic potential $V$, which models the adsorbate-substrate interaction at $T=0$; (2) a Gaussian white noise, $R_{G}(t)$, accounting for the lattice vibrational effects that the surface temperature induces on the adsorbate; and (3) a white shot noise, which stands for the adsorbate-adsorbate collisions and replaces the pairwise (dipole-dipole) interaction potential generally considered. In this way, a typical LMD simulation involving $N$ adsorbates is substituted by the dynamics of a single adsorbate, with the action of the remaining $N-1$ adsorbates being described by the random force associated with the white shot noise. This noise describes the adsorbate-adsorbate interaction by a series of random pulses within a Markovian regime (i.e., pulses of relatively short duration in comparison with the system relaxation). This interaction is, therefore, described in terms of a collisional friction which depends on the surface coverage. With this simple stochastic model, a better agreement with the experimental data for coverages up to around 20\%, approximately, is obtained. Although further investigation at microscopic level and calculations from first principles are needed, this simple stochastic model at moderate coverages is also able to provide a complementary view of diffusion and low frequency vibrational motions, described by the peaks at or around zero energy transfers (very long time dynamical processes), respectively. This could be understood because any trace of the true interaction potential seems to be 
wiped out due to the relatively large number of collisions taking place at very long times.

The organization of this paper is as follows. In Sec. II, we introduce the stochastic model used in the ISA approximation as well as its connection with the observable magnitudes in QHAS experiments. In Sec. III, the working model considered for the diffusion of $\mathrm{Na}$ on $\mathrm{Cu}(001)$ is described, and numerical results are presented and discussed. Final remarks and conclusions are summarized in Sec. IV.

\section{INTERACTING SINGLE ADSORBATE APPROXIMATION}

In analogy to scattering of slow neutrons by crystals and liquids, ${ }^{13,14}$ the observable magnitude in QHAS experiments is the differential reflection coefficient,

$$
\frac{d^{2} \mathcal{R}(\Delta \mathbf{K}, \omega)}{d \Omega d \omega}=n_{d} \mathcal{F} S(\Delta \mathbf{K}, \omega) .
$$

This coefficient gives the probability that the He atoms scattered from the adsorbates on the surface reach a certain solid angle $\Omega$ with an energy exchange $\hbar \omega=E_{f}-E_{i}$ and wave vector transfer parallel to the surface $\Delta \mathbf{K}=\mathbf{K}_{f}-\mathbf{K}_{i}$. In Eq. (1), $n_{d}$ is the concentration of adparticles; $\mathcal{F}$ is the atomic form factor, which depends on the interaction potential between the probe atoms in the beam and the adparticles on the surface; and $S(\Delta \mathbf{K}, \omega)$ is the dynamic structure factor or scattering law which gives the $Q$ and $T$ peaks, and it provides complete information about the dynamics and structure of the adsorbates through particle distribution functions. Experimental information about long distance correlations is obtained from the scattering law when considering small values of $\Delta \mathbf{K}$, while information on long time correlations is provided at small energy transfers, $\hbar \omega$.

The dynamic structure factor in Eq. (1) can also be expressed as

$$
S(\Delta \mathbf{K}, \omega)=\frac{1}{2 \pi} \int e^{-i \omega t} I(\Delta \mathbf{K}, t) d t,
$$

where

$$
\begin{aligned}
I(\Delta \mathbf{K}, t) & \equiv\langle\exp \{-i \Delta \mathbf{K} \cdot[\mathbf{R}(t)-\mathbf{R}(0)]\}\rangle \\
& =\left\langle\exp \left[-i \Delta K \int_{0}^{t} v_{\Delta \mathbf{K}}\left(t^{\prime}\right) d t^{\prime}\right]\right\rangle
\end{aligned}
$$

is the so-called intermediate scattering function. In this function, the brackets denote the ensemble average over trajectories $\mathbf{R}(t)$ running on the surface, and $v_{\Delta \mathbf{K}}$ is the velocity of the adparticle projected onto the direction of the parallel momentum transfer, $\Delta \mathbf{K}(\Delta K \equiv\|\Delta \mathbf{K}\|)$. Following the standard formulation used in neutron scattering theory, this function can be split into two contributions: $I_{s}$ (self-function), which corresponds to the average of trajectories of the same adparticle at two different times, and $I_{d}$ (distinct function), which corresponds to the average of trajectories of two different adparticles at different times. The Fourier transforms corresponding to $I$ and $I_{s}$ give what are called the coherent and incoherent scattering laws, $S(\Delta \mathbf{K}, \omega)$ and $S_{s}(\Delta \mathbf{K}, \omega)$, respectively.
In QHAS experiments, and with interacting adsorbates, coherent scattering is always obtained. The corresponding theoretical interpretation of this type of scattering is usually carried out in terms of Vineyard's convolution approximation, ${ }^{15}$ where the distinct pair correlation function is expressed as a convolution of the self pair correlation function. This approximation is known to fail at small distances, where the surface lattice becomes important. Whereas in neutron scattering many attempts to improve the convolution approximation have been developed, within the QHAS context, very little effort has been devoted to achieve this goal. At finite coverages, one usually distinguishes between two diffusion coefficients: ${ }^{16}$ the tracer diffusion constant $\left(D_{t}\right)$ and the collective diffusion constant $\left(D_{c}\right)$. $D_{t}$ refers to the selfdiffusion process and focuses on the motion of a single adsorbate. On the contrary, $D_{c}$ is related to the collective motion of all adsorbates, which is governed by Fick's law. In either case, a Kubo-Green formula relates $D_{t}$ or $D_{c}$ with the velocity autocorrelation function of a single adsorbate or with the corresponding for the velocity of the center of mass, respectively.

In the ISA approximation, the distinction between self and distinct time-dependent functions does not apply, and Eqs. (1) and (2) still hold. If the so-called Gaussian approximation ${ }^{16}$ is invoked, some analytical treatment is possible. This theoretical approach leads to a very simple and easy manner to interpret the underlying dynamics issued from the numerical Langevin simulations. Under this approximation, the intermediate scattering function can be expressed as a second-order cumulant expansion in $\Delta \mathbf{K}$ as

$$
I(\Delta \mathbf{K}, t) \approx \exp \left[-\Delta K^{2} \int_{0}^{t}\left(t-t^{\prime}\right) \mathcal{C}\left(t^{\prime}\right) d t^{\prime}\right] .
$$

In Eq. (4), $\mathcal{C}(t)$ is the velocity autocorrelation function, defined as

$$
\mathcal{C}(\tau) \equiv\langle v(0) v(\tau)\rangle
$$

along $\Delta \mathbf{K}$. The Gaussian approximation is exact when the velocity correlations at more than two different times are negligible, thus allowing to replace the average acting over the exponential function by an average acting over its argument.

The motion of an adsorbate under the action of a bath consisting of other adsorbates on a static two-dimensional general surface potential can be well described, in the Markovian approximation, ${ }^{7}$ by the standard Langevin equation

$$
\ddot{\mathbf{R}}(t)=-\eta \dot{\mathbf{R}}(t)+F[\mathbf{R}(t)]+\delta \mathbf{R}_{S}(t)+\delta \mathbf{R}_{G}(t),
$$

where $\mathbf{R}=(x, y)$ represents the surface lattice points and $\mathbf{F}$ $=-\nabla V$ is the deterministic force per mass unit, with $V(x, y)$ being the surface interaction potential with periods $a$ and $b$ along the $x$ and $y$ directions, respectively. The substrate excitations lead to the random force per mass unit $\mathbf{R}_{G}(t)$ on the adatom which has features of a Gaussian white noise. The shot noise $\mathbf{R}_{S}(t)$ is given by a series of impacts, and we have assumed sudden adsorbate-adsorbate collisions (i.e., strong but elastic) and after-collision effects relax exponentially. Moreover, the probability for collisions follows a Poisson 
distribution. No correlation has been assumed between both types of noise as well as between the noise sources associated with each degree of freedom.

In Eq. (6), $\eta=\gamma+\lambda$, where $\gamma$ is the frictional damping coefficient resulting from the nonadiabatic coupling to the electronic and vibrational excitations of the substrate, and $\lambda$ is the number of collisions per time unit or collisional friction. By using the elementary kinetic theory of transport in gases ${ }^{13}$ and the Chapman-Enskog theory for hard spheres, a simple relation can be found ${ }^{11}$ between the collisional friction coefficient $\lambda$ and the coverage $\theta$ at a temperature $T$,

$$
\lambda=\frac{6 \rho \theta}{a^{2}} \sqrt{\frac{k_{B} T}{m}},
$$

where $k_{B}$ is the Boltzmann constant, $a$ is the unit cell length of an assumed square surface lattice cell, and $\rho$ is the effective radius of an adparticle of mass $m$.

Finally, it is worth stressing the fact that, at long times, there will not be free diffusion because the effects of the two stochastic forces are dominant. This is the diffusive regime, where mean square displacements are linear with time,

$$
\left\langle x^{2}\right\rangle(t) \sim \frac{2 k_{B} T}{m \eta} t=2 D t,
$$

$D$ being the diffusion constant. In general, we can express Eq. (8) as

$$
D=\lim _{t \rightarrow \infty} \frac{1}{4 t}\left\langle|\mathbf{R}(t)-\mathbf{R}(0)|^{2}\right\rangle,
$$

which is known as Einstein's law, the value $D=k_{B} T / m \eta$ for the diffusion coefficient being the Einstein relation. Note from Eq. (8) that $D$ increases by lowering the total friction $\eta$ and by increasing the surface temperature.

\section{RESULTS AND DISCUSSION}

The diffusion of $\mathrm{Na}$ atoms on the $\mathrm{Cu}(001)$ surface can be considered as a prototype in QHAS since a lot of experimental and theoretical work can be found in the literature. The coverage $\theta_{\mathrm{Na}}=1$ corresponds to one $\mathrm{Na}$ atom per $\mathrm{Cu}(001)$ surface atom ${ }^{3}$ or, equivalently, $\sigma=1.53 \times 10^{19} \mathrm{atom} / \mathrm{cm}^{2} ; a$ $=2.557 \AA$ is the unit cell length and $\rho=2 \AA$ has been used for the atomic radius of $\mathrm{Na}$. The surface friction we have considered in our simulations is taken from Ref. 2 to be $\gamma$ $=0.1 \omega_{0}=2.2049 \times 10^{-5}$, where $\omega_{0}$ is the harmonic frequency associated with the periodic surface potential. Frequencies and times are given here in atomic units. We are going to analyze four coverages for two surface temperatures: 200 and $300 \mathrm{~K}$. On the one hand, $\theta_{\mathrm{Na}}=0.028$ and 0.064 , where the single adsorbate regime holds; on the other hand, $\theta_{\mathrm{Na}}$ $=0.106$ and 0.18 , where the interaction between adsorbates cannot be ignored. These coverage values correspond to $\lambda$ $=3.34 \times 10^{-6} \quad\left(\eta=2.53 \times 10^{-5}\right), \quad \lambda=7.64 \times 10^{-6} \quad(\eta=2.98$ $\left.\times 10^{-5}\right), \quad \lambda=1.26 \times 10^{-6} \quad\left(\eta=3.46 \times 10^{-5}\right), \quad$ and $\quad \lambda=2.15$ $\times 10^{-5}\left(\eta=4.68 \times 10^{-5}\right)$, respectively.

For the adsorbate-substrate interaction potential $V$, in this work we have considered the nonseparable model proposed by Ellis et al., ${ }^{2}$ whose form is

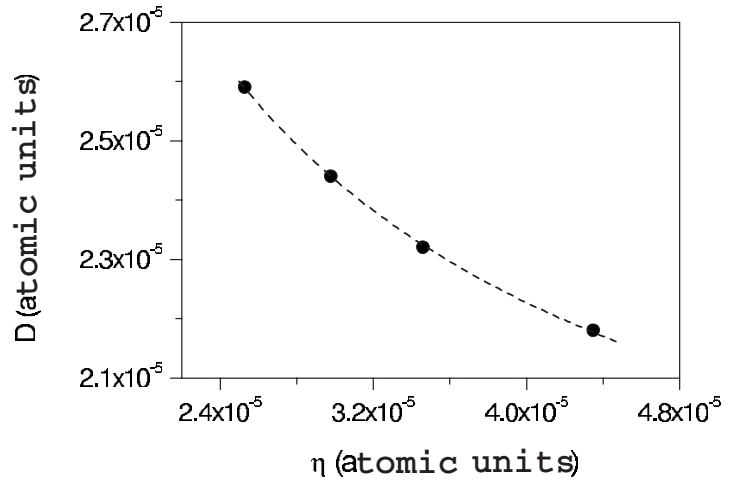

FIG. 1. Numerical (circles) and theoretical (dashed line) values for the diffusion coefficient $D$ as a function of the total friction $\eta$ at $T=200 \mathrm{~K}$ along the azimuth [100].

$$
V(x, y)=V_{0}(x, y)+V_{1}(x, y)+V_{2}(x, y) .
$$

In this expression, the first term is a simple separable cosine potential,

$$
V_{0}(x, y)=V_{0}[2-\cos (2 \pi x / a)-\cos (2 \pi y / a)],
$$

with $a$ the lattice constant of the $\mathrm{Cu}(001)$ surface and $V_{0}$ $=41.4 \mathrm{meV}$; the second term, which reads as

$$
V_{1}(x, y)=-A \sum_{m, n} e^{-b\left\{[(x / a)-(m+1 / 2)]^{2}+[(y / a)-(n+1 / 2)]^{2}\right\}},
$$

with $A=2 V_{0}$ and $b=11.8$, is added to produce a lowering of the potential barrier at on-top sites according to the observations; finally, the third term is also a nonseparable part which serves to alter the curvature near the minima and vary the difference between the potential at the minima and the bridge positions,

$$
\begin{aligned}
V_{2}(x, y)= & \pi^{2} C V_{0} \sum_{m, n}\left[\left(\frac{x}{a}-m\right)^{2}+\left(\frac{y}{a}-n\right)^{2}\right] \\
& \times \exp \left[-\left(\frac{x}{a}-m\right)^{2}-\left(\frac{y}{a}-n\right)^{2}\right],
\end{aligned}
$$

with $C=-0.2$. Two scattering azimuths, $[100]$ and $[1 \overline{1} 0]$, diagonal and parallel to the reciprocal space lattice in the first Brillouin zone, respectively, are considered here.

In the ISA approximation, Einstein's law, given by Eq. (9), is satisfied and, therefore, the diffusion constant $D$ decreases with the coverage. As said above, the origin of the friction arises from the collisions among adsorbates and the influence of the substrate phonons. This behavior is clearly observed in Fig. 1, where two types of calculations of the diffusion constant are plotted as a function of the total friction (and, therefore, of the coverage, since the surface friction coefficient is kept fixed in all our calculations) and for a surface temperature of $200 \mathrm{~K}$ along the azimuth [100]. The full circles correspond to the values obtained from Eq. (9) after performing the corresponding Langevin numerical simulation. The dashed line represents Einstein's relation with a fitting constant instead of the actual value $k_{B} T / \mathrm{m}$; the 

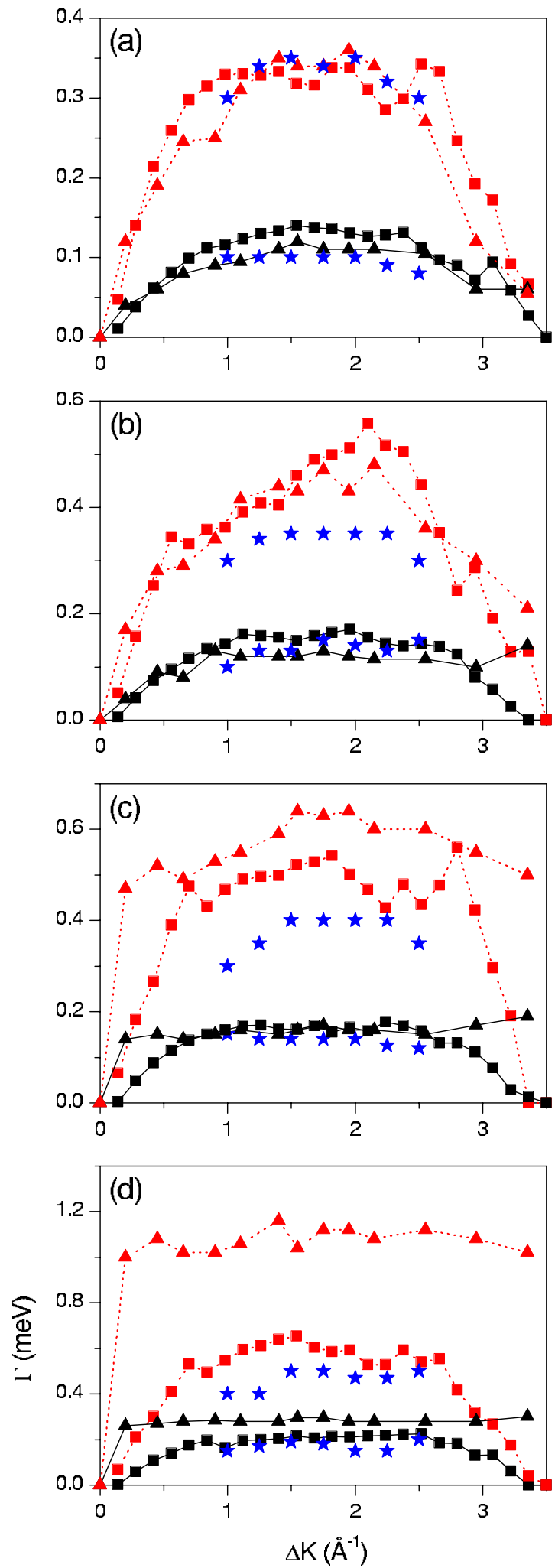

FIG. 2. (Color online) Numerical (squares) and experimental (triangles) dependence of $\Gamma$ on $\Delta K$ at $T=200 \mathrm{~K}$ [black (solid) line] and $T=300 \mathrm{~K}$ [red (dotted) line] along the azimuth [100]. Different values of the coverage are considered: (a) $\theta=0.028$, (b) $\theta=0.064$, (c) $\theta=0.106$, and (d) $\theta=0.18$. To compare with, in each case a few LMD results (blue stars) are also shown.
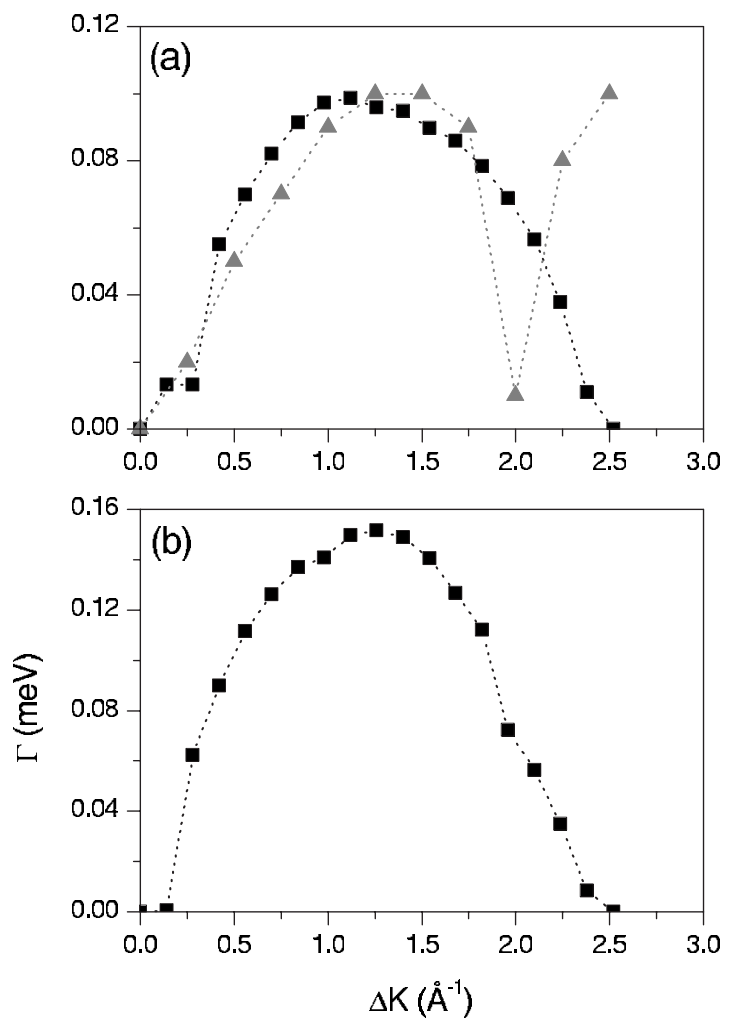

FIG. 3. Numerical (squares) and experimental (triangles) dependence of $\Gamma$ on $\Delta K$ at $T=200 \mathrm{~K}$ along the azimuth [1 $1 \overline{1} 0]$. Two values of the coverage are considered: (a) $\theta=0.028$ and (b) $\theta=0.18$.

value obtained for such a fitting constant would correspond to an effective mass 1.007 times the $\mathrm{Na}$ mass. As seen, the agreement is fairly good.

The broadening observed in the $Q$ peak with increasing coverage can be attributed ${ }^{10-12}$ to the respective fast decay undergone by the intermediate scattering function. The maximum value of the experimental peak widths is found to be increased by a factor of 3 as the coverage is increased from 0.028 to 0.18 . In Figs. 2(a)-2(d), the results of our model for the $Q$-peak width have been plotted and compared with the experimental ones for $\theta=0.028,0.064,0.106$, and 0.18 at $T$ $=200 \mathrm{~K}$ [black squares (solid lines) $]$ and $T=300 \mathrm{~K}$ [red squares (dotted lines)] together with the experimental ones (black and red triangles, respectively) along the azimuth [100]. To compare with, in each case a few LMD results are also shown (blue stars). As can be seen, LMD simulations reproduce the general trend, but predict a smaller increase in the broadening of the $Q$ peak. ${ }^{3}$ However, the agreement of the ISA results with the experiment is fairly good up to $\theta$ $=0.106$. This broadening has to be understood in terms of what we have termed as bound trajectories, ${ }^{10}$ associated with the motion of the adsorbates inside the potential wells: when adsorbates display this type of motion, the complex exponentials in Eq. (3) keep the coherence necessary for the intermediate scattering function to decay much slower than when the adsorbates are moving on the surface (running trajectories). This effect is directly related to the increment of $\eta$, which is equivalent to consider an increment of the collisional friction $\lambda$ (which increases with $\theta$ ), since we have chosen $\gamma$ as a 
constant substrate friction in all our numerical simulations. The same trend could be observed in the lineshapes associated with the $T$-mode peaks. ${ }^{10-12}$

As mentioned above, the agreement of our model with the experiment is at about $\theta=0.106$. It is clear then that this value should be regarded as a limit for our model. Note that with high coverages, the adsorbate-adsorbate interaction starts to play a more relevant role in the diffusion dynamics. The average motion of the adsorbate becomes slower and it feels the force exerted by its neighbors for longer times, which could end up in a sort of collective motion involving a number of adsorbates. This would give rise to a breakdown of the Markovian approximation and, therefore, taking into account memory effects.

Broadening with the coverage can also be observed along the $[1 \overline{1} 0]$ azimuth. As far as we know, no LMD simulations have been performed in this direction. Our numerical results (black squares) at two coverages $(\theta=0.028$ and 0.18$)$ and the experimental data (triangles) at $\theta=0.028$ are displayed in Figs. 3(a) and 3(b). In particular, the results for the highest value of the coverage are considered to be predictive.

\section{CONCLUSIONS}

A full but simple stochastic description of the lineshapes for activated surface diffusion and low-frequency vibrational motion for adsorbate/substrate systems with increasing coverages has been presented and discussed. A simple Langevin equation within the ISA approximation is used to calculate the adparticle dynamics, where a Gaussian white noise accounting for the effect of the adsorbate-substrate friction and a white shot noise simulating the friction due to the collisions among adsorbates, with no correlation between them, are considered to describe microscopic interactions. In our opinion, the present numerical results for the broadening of the $Q$ peak, which are obtained with no fitting parameter, are in better agreement than previous LMD simulations, mainly due to the role of the collisional friction introduced in our stochastic model. We are well aware that the barrier diffusion should be modified with the coverage. ${ }^{3}$ However, our goal here was only to study the collisional friction effects. Obviously, this type of study cannot replace further investigations at microscopic level, and calculations from first principles of this dynamics need to be done. The complementary view we propose in this work should remain valid because the lineshapes of the diffusion and low-vibrational-frequency motion analyzed at very long times, where the number of collisions is relatively large (or where the statistical limit is applicable), seem to wipe out any trace of microscopic interactions.

\section{ACKNOWLEDGMENTS}

This work was supported in part by DGCYT (Spain) under Project No. FIS2007-62006. R.M.-C. would like to thank the University of Bochum for support from the Deutsche Forschungsgemeinschaft, SFB 558. A.S.S. would like to thank the Spanish Ministry of Education and Science for a "Juan de la Cierva" contract. *ruth@imaff.cfmac.csic.es

†jlvega@imaff.cfmac.csic.es

†asanz@imaff.cfmac.csic.es

\$s.miret@imaff.cfmac.csic.es

${ }^{1}$ S. Miret-Artés and E. Pollak, J. Phys.: Condens. Matter 17, S4133 (2005).

${ }^{2}$ A. P. Graham, F. Hofmann, J. P. Toennies, L. Y. Chen, and S. C. Ying, Phys. Rev. B 56, 10567 (1997).

${ }^{3}$ J. Ellis, A. P. Graham, F. Hofmann, and J. P. Toennies, Phys. Rev. B 63, 195408 (2001).

${ }^{4}$ J. L. Vega, R. Guantes, and S. Miret-Artés, J. Phys.: Condens. Matter 14, 6193 (2002); 16, S2879 (2004).

${ }^{5}$ J. L. Vega, R. Guantes, and S. Miret-Artés, Phys. Chem. Chem. Phys. 4, 4985 (2002); R. Guantes, J. L. Vega, S. Miret-Artés, and E. Pollak, J. Chem. Phys. 119, 2780 (2003).

${ }^{6}$ R. Guantes, J. L. Vega, S. Miret-Artés, and E. Pollak, J. Chem. Phys. 120, 10768 (2004); J. L. Vega, R. Guantes, S. MiretArtés, and D. A. Micha, ibid. 121, 8580 (2004).
${ }^{7}$ R. Martínez-Casado, J. L. Vega, A. S. Sanz, and S. Miret-Artés, J. Phys.: Condens. Matter 19, 176006 (2007).

${ }^{8}$ R. Martínez-Casado, J. L. Vega, A. S. Sanz, and S. Miret-Artés, J. Chem. Phys. 126, 194711 (2007).

${ }^{9}$ G. Alexandrowicz, A. P. Jardine, H. Hedgeland, W. Allison, and J. Ellis, Phys. Rev. Lett. 97, 156103 (2006).

${ }^{10}$ R. Martínez-Casado, J. L. Vega, A. S. Sanz, and S. Miret-Artés, Phys. Rev. Lett. 98, 216102 (2007).

${ }^{11}$ R. Martínez-Casado, J. L. Vega, A. S. Sanz, and S. Miret-Artés, Phys. Rev. E 75, 051128 (2007).

${ }^{12}$ R. Martínez-Casado, J. L. Vega, A. S. Sanz, and S. Miret-Artés, J. Phys.: Condens. Matter 19, 305002 (2007).

${ }^{13}$ D. A. McQuarrie, Statistical Mechanics (Harper and Row, New York, 1976).

${ }^{14}$ L. van Hove, Phys. Rev. 95, 249 (1954).

${ }^{15}$ G. H. Vineyard, Phys. Rev. 110, 999 (1958).

${ }^{16}$ R. Gomer, Rep. Prog. Phys. 53, 917 (1990). 